JIANGFENG WANG, Ph. D. ${ }^{1}$

(Corresponding author)

E-mail: wangjiangfeng@bjtu.edu.cn

CHANG GAO, M.S. ${ }^{2}$

Email: 14125692@bjtu.edu.cn

ZHOUYUAN ZHU, M.S. ${ }^{2}$

E-mail: 14125756@bjtu.edu.cn

XUEDONG YAN, Prof. $^{1}$

E-mail: xdyan@bjtu.edu.cn

${ }^{1}$ Beijing Jiaotong University, MOE Key Laboratory for Urban

Transportation Complex Systems Theory and Technology

3 Shang Yuan Cun, Hai Dian District,

Beijing 100044, P.R. China

2 Beijing Jiaotong University, School of Traffic

and Transportation, 3 Shang Yuan Cun,

Hai Dian District, Beijing 100044, P.R. China
Human - Transport Interaction

Preliminary Communication

Submitted: 9 Mar. 2016

Accepted: 16 Nov. 2016

\title{
MULTI-LANE CHANGING MODEL WITH COUPLING DRIVING INTENTION AND INCLINATION
}

\begin{abstract}
Considering the impact of drivers' psychology and behaviour, a multi-lane changing model coupling driving intention and inclination is proposed by introducing two quantitative indices of intention: strength of lane changing and risk factor. According to the psychological and behavioural characteristics of aggressive drivers and conservative drivers, the safety conditions for lane changing are designed respectively. The numerical simulations show that the proposed model is suitable for describing the traffic flow with frequent lane changing, which is more consistent with the driving behaviour of drivers in China. Compared with symmetric two-lane cellular automata (STCA) model, the proposed model can improve the average speed of vehicles by $1.04 \%$ under different traffic demands when aggressive drivers are in a higher proportion (the threshold of risk factor is 0.4). When the risk factor increases, the average speed shows the polarization phenomenon with the average speed slowing down in big traffic demand. The proposed model can reflect the relationship among density, flow, and speed, and the risk factor has a significant impact on density and flow.
\end{abstract}

\section{KEY WORDS}

lane changing model; driver intention; driving inclination; cellular automaton;

\section{INTRODUCTION}

Vehicle lane changing can bring vital influence to the efficiency and safety of the entire traffic flow. It also leads to speed variance and flow instability that will directly cause traffic accidents. Vehicle lane changing contributes to about $4 \%$ to $10 \%$ of traffic accidents, of which about $75 \%$ are caused by human factors. In addition, about $10 \%$ of traffic delays are caused by lane changing, bringing about huge economic losses to the society, and irreparable negative effect to the ecological environment [1].

Drivers' psychological activities and behaviours are strongly stochastic in lane changing process and result in the randomness and complexity of traffic flow [2]. To reveal the influence mechanism of lane changing behaviour on the efficiency and safety of the entire traffic flow, scholars at home and abroad have been attempting to study the effect of the law of drivers' psychology and behaviour in lane changing process, and have proposed many improved models which are suitable for weaving sections impact analysis [3], access control strategy [4], lane changing trajectory [5] and crash risk reduction [6]. The contributions of these studies focus on the analysis and modelling of lane changing behaviours in specific areas using traffic flow theory. In terms of research means, Hoseini uses probability theory, and Cellular Automaton (CA) to analyse the impact of lane changing behaviour on the traffic flow [7]. The results indicate that the model based on driving regulations is not valid, but that three probabilistic models appear useful for predicting the driver's behaviour on freeway segments during non-congested conditions. CA has some advantages such as non-linear simulation, flexible calculation and reproduction of a variety of real traffic conditions, etc. It is a classic method for studying lane changing behaviour [8, 9, 10, $11,12]$. The first use of stochastic cellular automata for traffic flow was introduced by Nagel and Schreckenberg, referred as the NaSch model [13]. Most of the former CA lane changing models have been optimized and improved based on the NaSch model. The symmetric two-lane cellular automata (STCA) model, STCA extended model, lane changing model based on fuzzy 
control and urban lane changing model are presented based on the CA model according to the actual traffic flow and corresponding lane changing rules [14, 15]. The STCA model is proposed to descript the flow in two lanes, and can simulate the lane changing behaviour [16-19]. However, the drivers' lane changing intention and inclination are neglected in these models.

Different drivers have obviously different behaviours, thus rendering randomness and complexity to the traffic flow. However, the existing studies cannot reflect the implicit difference in driving behaviour in the real traffic flow [20]. Due to the importance of driver's lane changing intention and behaviour, many scholars conduct studies about recognizing lane changing intention [21], and apply the drivers' behavioural preferences [22], cooperation intention [23, 24] and sensitivity factor $[25,26]$ to design the lane changing model. In these studies, the driver characteristics are introduced into the lane changing model. Besides, common simulation software, such as VISSIM, also provide several parameters, such as expected speed to reflect qualitatively the impact of drivers' characteristics; however, it lacks quantitative parameters. Based on the analysis of lane changing requirements, two quantitative characteristic variables are introduced in this paper; namely, the intention strength of lane changing and the risk factor, which describe the driver's psychology and behaviour. A multi-lane changing model is also proposed, known as the Coupling Driving Intentions and Inclination (C-Dlal) model. C-Dlal model is used to simulate traffic flow under different traffic demands and to analyse the differences and reasons of traffic flow under various risk factors.

\section{MULTI-LANE CHANGING MODEL}

\subsection{Analysis of lane changing behaviour}

Driving behaviour can be classified as car following model and lane changing model. Lane changing model is an integrated process based on the driver's judgment and adjustment of the information of the surrounding environment, which is affected by the driver's spatial-temporal perception characteristics. The lane changing intention will be produced when the leading vehicle status does not meet the driver's expectations. To have a comfortable driving environment, a driver will undertake the lane changing behaviour coupling their driving inclination.

Generally, the driver type $\left(D_{T}\right)$ can be divided into conservative type $\left(D_{T}=0\right)$ and aggressive type $\left(D_{T}=1\right)$. Conservative drivers tend to slow down and follow the leading vehicle when traffic is congested, and choose to change lanes only when changing conditions are good enough, while aggressive drivers tend to change lanes to keep the expected speed once the conditions are satisfied.

Lane changing does not depend only on the vehicle status of the current lane and the adjacent lane, but it also depends on the driving inclination and the perception of the space resources around, and other factors. The lane changing intention and implementation are affected by the driver's perception of the road traffic, lane change constraints and risk acceptance. The decision-making process and the influencing factors of lane changing are shown in Figure 1.

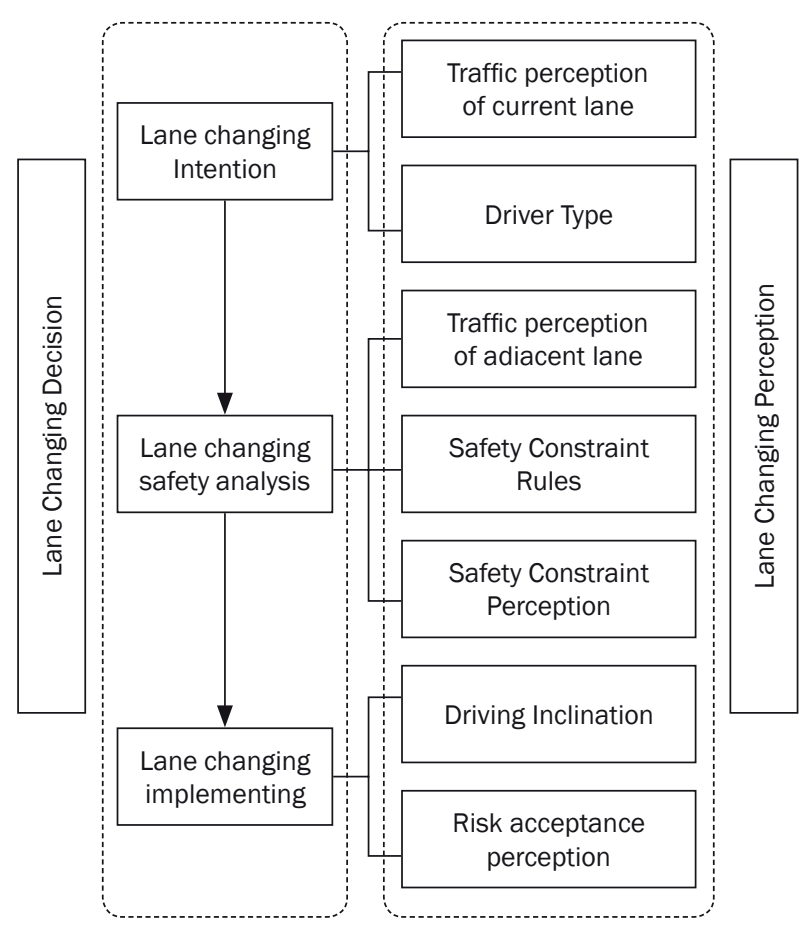

Figure 1 - Decision-making process and influencing factors of lane changing

In this paper, Multi-lane changing model is studied by taking free lane changing as the research object considering lane changing intention and driving inclination. On the basis of NS model, a lane changing model suitable for more realistic driving environment is proposed, and the impact of vehicle lane changing on traffic flow efficiency in different traffic demand is also analysed.

\subsection{Model design}

Before introducing the C-Dlal model, the classical NaSch model is briefly recalled [13]. The NaSch model is a discrete model for the traffic flow. The road is divided into cells, which can be either empty or occupied by a vehicle with a velocity $v=0,1, \ldots, v_{\max }$. The vehicles which are numbered $1,2, \ldots, i$ move from the left to the right on a lane with periodic boundary conditions. 
At each discrete time step $t \rightarrow t+1$ the system update is performed in parallel according to the following four rules:

Step 1: acceleration, $v(i) \rightarrow \min \left(v_{\max }, v(i)+1\right)$;

Step 2: deceleration, $v(i) \rightarrow \min (v(i), d(i))$;

Step 3: randomization, $v(i) \rightarrow \max (v(i)-1,0)$ with probability $p$;

Step 4: position update, $x(i) \rightarrow x(i)+v(i)$.

Here $v(i)$ and $x(i)$ denote the velocity and position of vehicle $i$, respectively; $v_{\max }$ is the maximum velocity and $d(i)=x(i-1)-x(i)-1$ denotes the number of empty cells in front of vehicle $i ; p$ is the randomization probability.

This set of rules controls the forward motion of vehicles. For the multi-lane traffic, the set of rules includes lane changing rules and car following rules. Thus, in multi-lane models the update step is usually divided into two sub-steps. In the first sub-step, vehicles may change lanes in parallel according to lane changing rules and in the second sub-step the lanes are considered as independent single-lane NaSch models.

The lane changing model describes the lane changing decision-making process of different types of drivers with lane changing intention. Considering the travelling condition of the current lane and the adjacent lane, a driver may have lane changing intention when the leading vehicle restricted their driving. Therefore, the lane changing intention factor $\alpha$ is introduced to characterize the intention strength of lane changing.

$\alpha(i)=\frac{T \cdot \min \left\{v(i)+1, v_{\max }\right\}}{d(i)}$

where $\alpha(i)$ is the intention strength of lane changing of the $i$-th driver; $\alpha(i)>1$ means the existence of lane changing intention, and the larger $\alpha(i)$ value indicates a stronger intention strength of lane changing; $T$ is the time step, $\mathrm{s}$, and equals $1 \mathrm{~s}$ in this paper; $v(i)$ is the speed of the $i$-th vehicle, $\mathrm{m} / \mathrm{s} ; v_{\max }$ is the maximal speed, $\mathrm{m} / \mathrm{s} ; d(i)$ is the distance between the $i$-th vehicle and the leading vehicle of the current lane, $m$.

When lane changing intention occurs, whether the lane changing manoeuvre is eventually implemented depends on the driver's lane changing inclination and the safety condition. The lane changing inclination depends directly on the driver's character, because different types of drivers have different tendencies of lane changing. The concept of the driver's risk factor $\beta$, which indicates the intensity of lane changing inclination, is introduced to represent the lane changing inclination of different types of drivers. A bigger $\beta$ value represents more probability of lane changing. The value is set as $\beta \in(0,1)$. In the simulation process, $\beta$ is determined by random numbers, and the driver type of each vehicle can be calculated by Formula 2 .

$D_{T}= \begin{cases}0 & 0<\beta \leq \beta_{T} \\ 1 & \beta_{T}<\beta<1\end{cases}$ where $\beta_{T}$ is the threshold of driver type, $\beta \in(0,1)$.

Drivers would show lane changing inclination when their vehicles are restrained by the leading vehicle. The lane changing behaviour of different types of drivers depends on their driving inclination, and the lane changing safety conditions should be satisfied according to their corresponding driving behaviour. Conservative drivers may only change a lane when the constraints of lane changing are relatively loose. Considering the intention strength $\alpha(\mathrm{i})$, the lane changing conditions of conservative drivers can be indicated by Formula 3.

$$
\left\{\begin{array}{l}
\alpha(i)>1 \\
d(i)<\min \left\{\operatorname{int}\left(\alpha \cdot v(i) \cdot T, v_{\max } \cdot T\right\}\right. \\
d(i, I v)>d(i) \\
d(i, f v)>v_{\max } \cdot T
\end{array}\right.
$$

where int() is integral function; $d(i, I v), d(i, f v)$ are the distance between the $i$-th vehicle and the leading vehicle of adjacent lane, the distance between the $i$-th vehicle and the followings vehicle of adjacent lane, $\mathrm{m}$.

The aggressive drivers, who have a stronger intention of lane changing, and are more likely to undertake frequent lane changing to satisfy their intention in a higher risk factor $\beta$. Hence, the constraints of lane changing of aggressive drivers are relatively stricter than those of conservative drivers. According to reference [27], the lane changing conditions can be indicated by Formula 4.

$$
\left\{\begin{array}{l}
\alpha(i) \geq 1 \\
d(i)<T \cdot \min \left\{v(i)+1, v_{\max }\right\} \\
d(i, I v)>d(i) \\
d(i, f v)>1+T \cdot \min \left\{v(i, f v)+1, v_{\max }-\right. \\
\quad-T \cdot \min \left\{v(i)+1, v_{\max }\right\}
\end{array}\right.
$$

Therefore, C-Dlal model is proposed as follows, considering lane changing intention and the characteristic of driving inclination of different types of drivers.

$C=\left\{\begin{array}{cc}1 & D_{T}=0 \text { and Eq.3 } \\ 1 & D_{T}=1 \text { and Eq.4 } \\ 0 & \text { other }\end{array}\right.$

where $C$ is the lane changing decision variable, $C=1$ means changing lane according to lane changing logic priority rule; $\mathrm{C}=0$ means continue following the leading vehicle by using NS model.

A logical flow chart of the model simulation is provided in Figure 2, and the C-Dlal model is validated.

Lane changing logic priority rule should be considered in multi-lane $(n>2, n=3$ in this paper) traffic flow. It involves the position of vehicles and the lane changing order between different vehicles with lane changing intention. Several typical lane-changing scenarios are shown in Figure 3.

In Figure 3, lane $L_{1}$ is the outermost lane, and lane $\mathrm{L}_{3}$ is the innermost lane. According to the above three 


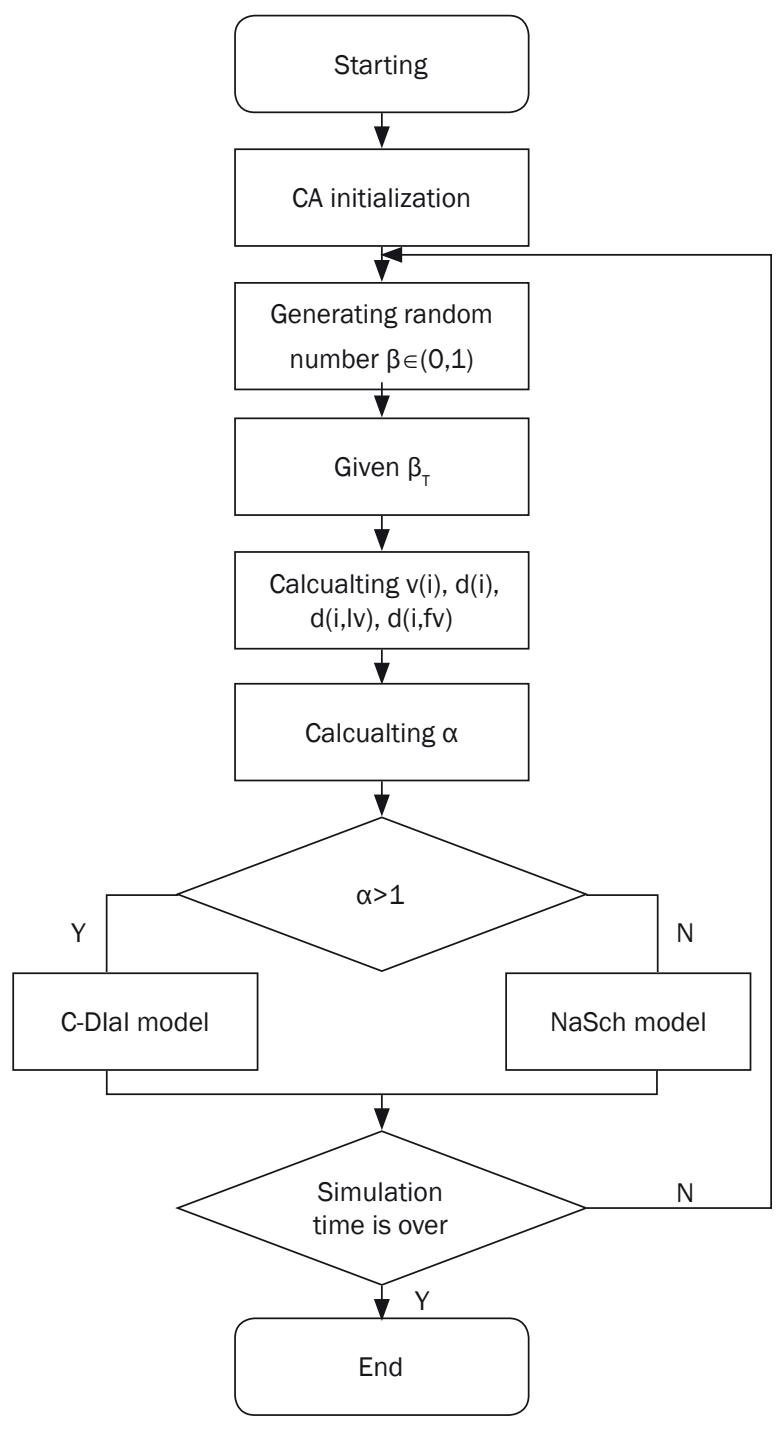

Figure 2 - Logic flow chart of model simulation scenarios, the following lane changing logic rules are set combining with reasonable changing conditions:

Rule 1: In scenario a), vehicle $\mathrm{V}_{\mathrm{s}}$ with changing intention can change lane from lane 1 to lane 2. However, $V_{2}$ in lane $L_{2}$ and $V_{3}$ in lane $L_{3}$ can change the lane first, if they also have lane changing intention.

Rule 2: in scenario $\mathrm{b}$ ), vehicle $\mathrm{V}_{\mathrm{s}}$ with changing intention can change to either lane $L_{1}$ or lane $L_{3}$, and it will switch to lane $L_{3}$ first. However, $V_{3}$ in lane $L_{3}$ can change first, if it also has lane changing intention.

Rule 3: in scenario $\mathrm{c}$ ), vehicle $\mathrm{V}_{\mathrm{s}}$ with changing intention can change lane from lane $L_{3}$ to lane $L_{2}$. It still can change first, even if $V_{2}$ in lane $L_{2}$ and $V_{1}$ in lane $L_{1}$ also have lane changing intention.

\section{NUMERICAL SIMULATION}

To verify the efficiency of the model, an experimental scheme for three lanes has been designed. Lane changing intention strength $\alpha$ and risk factor $\beta$ are used as independent variables to analyse the effect of the C-Dlal model on the traffic flow. With classical STCA model as reference, a comparative analysis was made of the effect on traffic flow under different traffic demands.

The settings of parameters in the simulation experiment are shown in Table 1.

\subsection{Lane changing frequency under different traffic demands}

Given a certain proportion of driver type $\left(\beta_{T}=0.4\right)$, the cumulative lane changing frequency under different traffic demands of two models is shown in Figure 4.

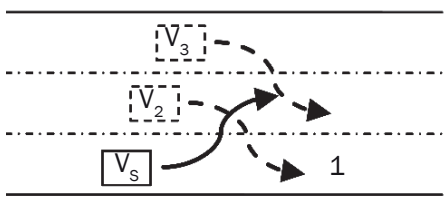

a)

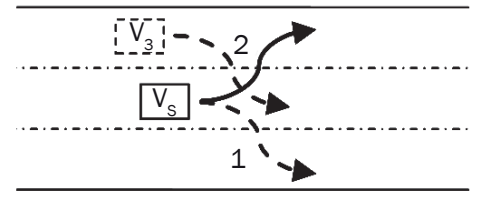

b)

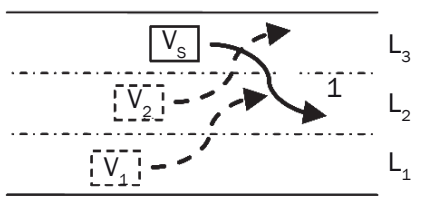

c)

Figure 3 - Schematic diagram of multi-lane changing logic priority rules

Table 1 - Parameter settings

\begin{tabular}{||l|l|l|l||}
\hline \multicolumn{1}{|c|}{ Parameters } & \multicolumn{1}{c|}{ Value } & \multicolumn{1}{c||}{ Parameters } & \multicolumn{1}{c||}{ Value } \\
\hline \hline Lane length $[\mathrm{m}]$ & 1,500 & Risk factor $\beta$ & $0.2 / 0.4 / 0.6 / 0.8$ \\
\hline The number of lanes & 3 & Vehicle arriving & Poisson distribution \\
\hline Cellular length $[\mathrm{m}]$ & 7.5 & Iterative times & 1,000 \\
\hline Maximum speed $[\mathrm{m} / \mathrm{s}]$ & 22.5 & Iteration step length/s & 1 \\
\hline Traffic demand $[\mathrm{pcu} / \mathrm{h} / \mathrm{In}]$ & $200 / 300 / 400 / 500 / 600$ & Randomization probability & 0.3 \\
\hline
\end{tabular}




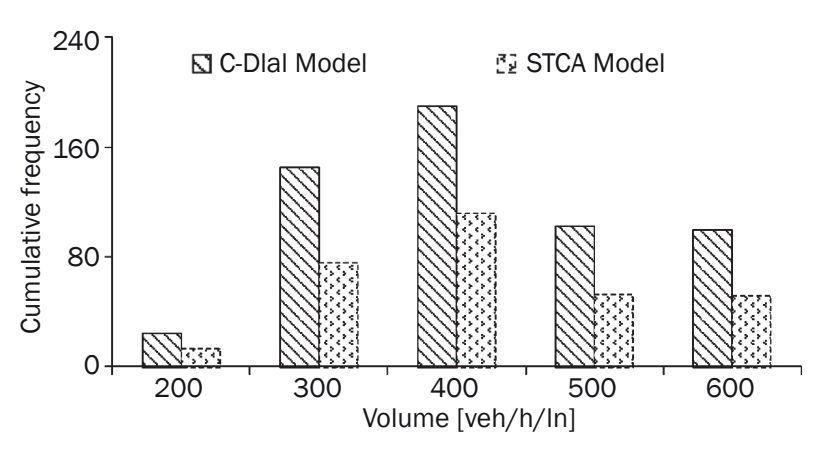

Figure 4 - Accumulative number of lane changing

As shown in Figure 4, with the increase of the input volume, the cumulative lane changing frequency of two models shows a trend of increasing first and then decreasing. This is because there is enough space to change lane when the input flow is small, and the lane changing demand would be constantly increased if the lane changing conditions were satisfied. When the input volume exceeds $400 \mathrm{veh} / \mathrm{h} / \mathrm{In}$, the lane changing space available on the road will decrease, and more vehicles in the target lane will be obstructed by the lane changing manoeuvre. Then the probability of satisfying the lane changing condition decreases and the lane changing demand is inhibited and decreased accordingly. When $\beta_{T}=0.4$, a larger proportion of vehicles have a strong inclination to change lane as for the large number of aggressive drivers. For the same input volume, there is more frequency of lane changing in C-Dlal model compared with STCA model.

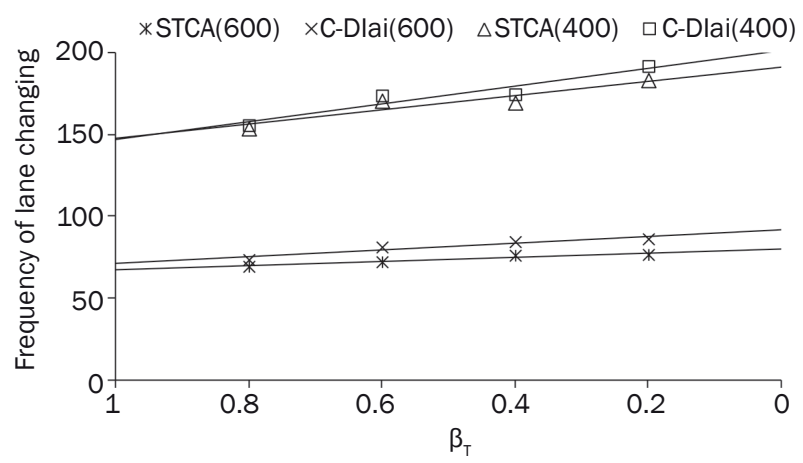

Figure 5 - Impact of risk factors on the number of lane changing

In order to explore the impact of different types of drivers on the lane changing manoeuvre, the interaction between risk factor $\beta$ and lane changing frequency is analysed. Figure 5 shows the impact of risk factor $\beta$ on lane changing frequency corresponding to input volume of $400 \mathrm{veh} / \mathrm{h} / \mathrm{In}$ and $600 \mathrm{veh} / \mathrm{h} /$ In. The result shows that the lane changing frequency of C-Dlal model is bigger compared with STCA model. Lane changing frequency of two models shows a rapid growth when the input volume is $400 \mathrm{veh} / \mathrm{h} / \mathrm{In}$, and C-Dlal model has a more obvious increasing trend. The lane changing frequency of two models has a slow growth when the input volume is $600 \mathrm{veh} / \mathrm{h} / \mathrm{ln}$ because of the limit of changing space.

\subsection{Variety of traffic flow parameters in different traffic demands}

Given $\beta_{T}=0.4$, the average speed distribution of two models in different input flows is shown in Figure 6.

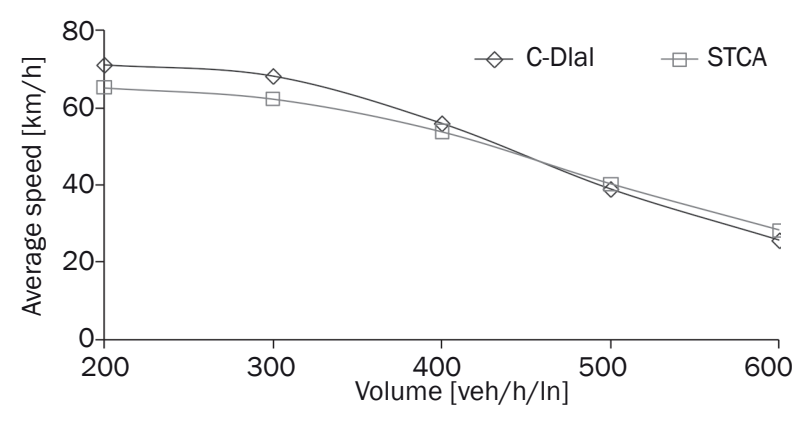

Figure 6 - Distribution of average speed with different flows

As shown in Figure 6, with the increase of the input flow, the average speed of the traffic flow showed a decreasing trend. C-Dlal model considers driving intention and inclination, so that more vehicles can change lane to increase vehicle speed when $\beta_{T}=0.4$; therefore, the higher speed can be reached under C-Dlal model with the same input volume. The comparison of the average speed of two models under different traffic demands is shown in Table 2. It can be seen from Table 2 that the average speed of the C-Dlal model has savings according to that of the STCA model when the input volume is relatively small ( $\leq 400 \mathrm{veh} / \mathrm{h} / \mathrm{In})$. This can be explained by the reasons that more aggressive drivers would change lane to achieve a higher speed; therefore, a significant improvement in the average speed is present under the C-Dlal model. When the input volume exceeds a certain value (>400 veh/h/In), though lane changing space

Table 2 - Comparison of the average speed of two models with different flows

\begin{tabular}{|c|c|c|c|c|c|c|c|c|c|c|}
\hline \multirow{3}{*}{$\begin{array}{l}\text { Model } \\
\text { STCA }\end{array}$} & \multicolumn{10}{|c|}{ Average speed [km/h] } \\
\hline & \multicolumn{2}{|c|}{$q=200 \mathrm{veh} / \mathrm{h} / \mathrm{ln}$} & \multicolumn{2}{|c|}{$q=300 \mathrm{veh} / \mathrm{h} / \mathrm{In}$} & \multicolumn{2}{|c|}{$q=400 \mathrm{veh} / \mathrm{h} / \mathrm{In}$} & \multicolumn{2}{|c|}{$q=500 \mathrm{veh} / \mathrm{h} / \mathrm{ln}$} & \multicolumn{2}{|c|}{$q=600 \mathrm{veh} / \mathrm{h} / \mathrm{In}$} \\
\hline & 65.29 & \multirow{2}{*}{$8.07 \% \uparrow$} & 63.38 & \multirow{2}{*}{$6.96 \% \uparrow$} & 53.85 & \multirow{2}{*}{$3.65 \uparrow$} & 40.29 & \multirow{2}{*}{$-3.52 \downarrow$} & 28.21 & \multirow{2}{*}{$-9.94 \downarrow$} \\
\hline C-Dlal & 71.02 & & 68.12 & & 55.89 & & 38.92 & & 25.66 & \\
\hline Savings & \multicolumn{10}{|c|}{$1.04 \% \uparrow$} \\
\hline
\end{tabular}


available is limited, a majority of aggressive drivers would still change lane when the changing conditions are satisfied. Therefore, a decline tendency of average speed is present under the C-Dlal model. According to the above analysis, the average speed of the C-Dlal model will be lower than that of the STCA model when the input volume is at a certain value between $400 \mathrm{veh} / \mathrm{h} / \mathrm{In}$ and $500 \mathrm{veh} / \mathrm{h} / \mathrm{In}$. In general, the efficiency of the entire traffic flow is improved as the average speed of the C-Dlal model features an increase of 1.04\% compared with the STCA model.

The impact of the proportion of different types of drivers on the traffic flow is analysed further in the text.

Figure 7 shows the impact of $\beta_{T}$ on the average speed under different traffic demands.

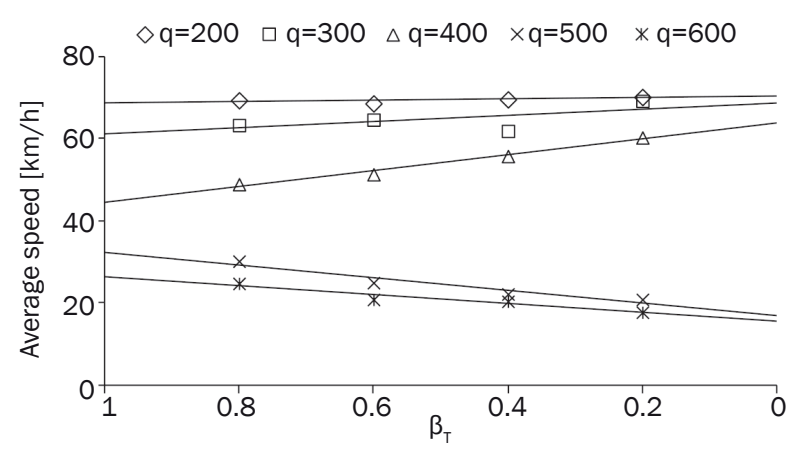

Figure 7 - Impact of risk factors on the average speed

As shown in Figure 7, the average speed of the C-DIal model shows a polarization trend with the change of $\beta_{T}$ value in different input volumes. With the decrease of the $\beta_{T}$ value, namely, the increase of the proportion of aggressive drivers, the average speed of the C-Dlal model shows an increasing tendency when the input volume is relatively small ( $\leq 400 \mathrm{veh} / \mathrm{h} / \mathrm{In}$ ). However, the average speed shows an opposite trend when the input volume is relatively high ( $>400 \mathrm{veh} / \mathrm{h} / \mathrm{In})$. The results indicate that the lane changing manoeuvre will have a different effect on the traffic flow with the increase of the proportion of aggressive drivers, which is also consistent with the common sense of actual traffic flow. The space for lane changing is relatively adequate under low traffic demand. Once a vehicle is obstructed by the leading vehicle, an aggressive driver would immediately change lane to seek a better driving environment if the lane changing condition is satisfied, which improves the flow efficiency. With the increase of traffic demand, the lane changing would seriously affect rear vehicles under high traffic demand for the shortage of lane changing space. Rear vehicles must take urgent braking to avoid an accident, which causes deterioration of the traffic flow and a decrease of the average speed of the entire traffic flow.

In order to explore the impact of risk factor on the traffic flow, the relationship between density and volume in different risk factors $\beta_{T}$ is analysed, as shown in Figure 8.

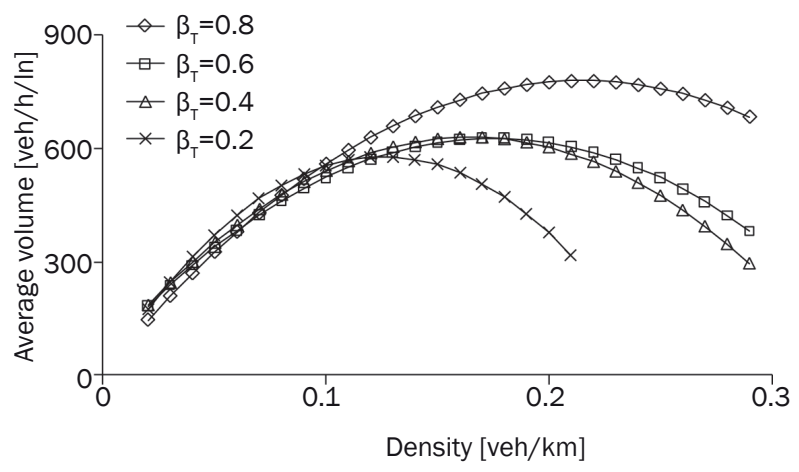

Figure 8 - Impact of risk factor on the relationship between density and volume

As shown in Figure 8, the relationship between density and volume could be reflected correctly by the C-Dlal model, and different risk factors would also affect their relationships. For low density, the impact of risk factor on the relationship between density and volume is not obvious. However, the impact is obvious for the case of high density. With the same density, a higher proportion of aggressive drivers means lower average volume of the C-Dlal model. It is reasonable that less lane changing space available in high density results in higher proportion of aggressive drivers changing lane. It would lead to serious interference on the traffic flow and reduce the average volume by the traffic congestion.

The relationship between density and speed of the C-Dlal model at different $\beta_{T}$ values is shown in Figure 9. The relationship between density and speed could be reflected correctly by the $\mathrm{C}$-Dlal model, and the impact of different risk factors on the relationship between density and speed is not obvious.

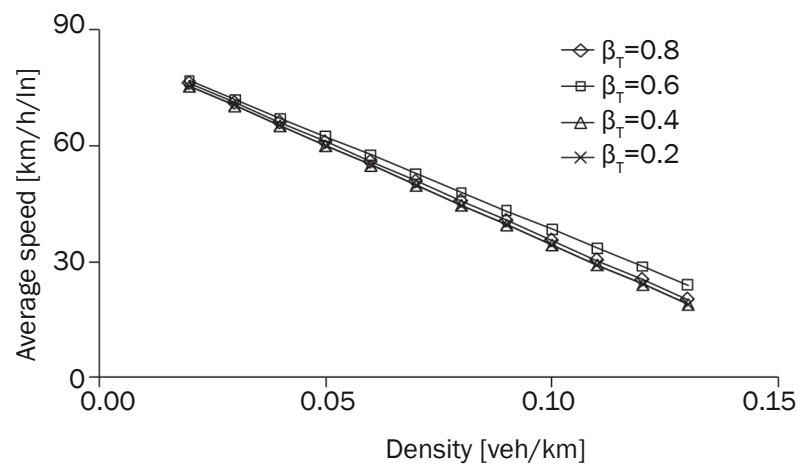

Figure 9 - Impact of risk factor on the relationship between density and speed

\subsection{Space-time diagrams analysis}

Space-time diagrams of two models with different volumes (200 veh/h/In, 400 veh/h/In, 600 veh/h/In) is shown in Figure 10. The horizontal axis represents space while the vertical axis represents time. Vehicles 


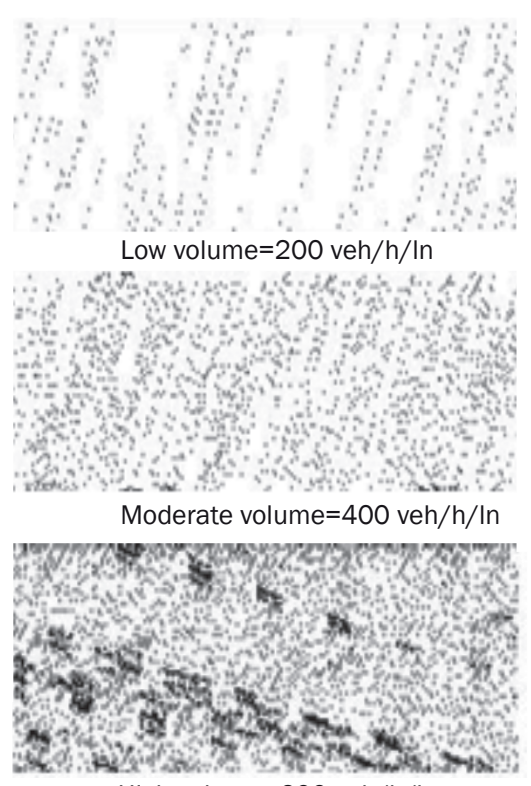

High volume $=600 \mathrm{veh} / \mathrm{h} / \mathrm{ln}$ a) STCA

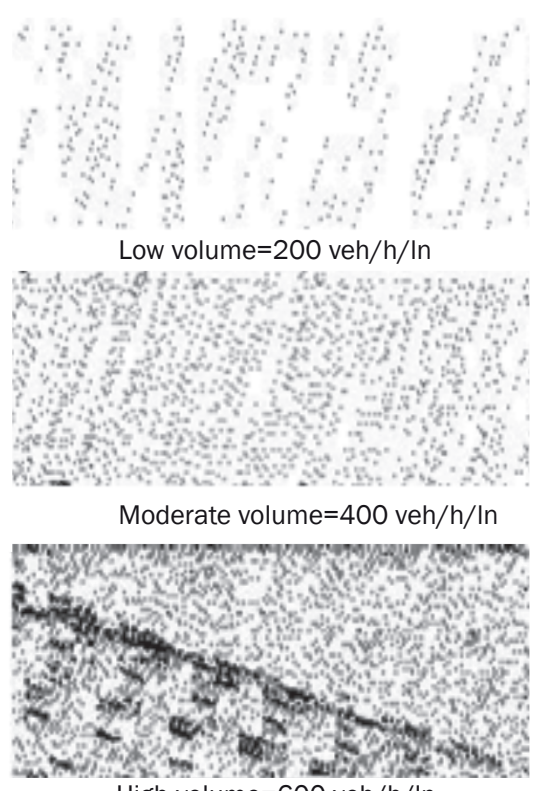

High volume $=600 \mathrm{veh} / \mathrm{h} / \mathrm{In}$

b) C-Dlal

Figure 10 - Space-time diagrams of two models with different flows

move from left to right in space axis, while from top to bottom in time axis. Given $\beta_{T}=0.4$, the C-Dlal model would change lane more frequently in case of high input flow (600 veh/h/In), and cause traffic congestion on the road. Excessive lane changing would affect the running efficiency of the entire traffic flow to some extent. Therefore, the aggravation of the traffic conditions is shown in the entire traffic flow.

\section{CONCLUSIONS AND DISCUSSION}

By coupling the driving intention and inclination, the C-Dlal model is presented considering the impact of the driver's character on lane changing. Numerical analysis results show that frequency of lane changing is related to the proportion of different types of drivers. Generally, there is a positive correlation between risk factor and traffic flow efficiency in lower traffic demand. Lane changing behaviour is helpful to improve the traffic efficiency. However, frequent lane changing will have a negative impact on the traffic efficiency under higher traffic demand, because it will decrease the vehicle average speed. Compared with the STCA model, the C-Dlal model is suitable for representing the traffic flow with high lane changing proportion, which is more consistent with the driver's frequent lane changing behaviour in China. This study will provide a reference for the design of multi-lane changing $C A$ model from the driver's character perspectives.

Vehicles in simulation are considered as the standard passenger cars. However, there are different types of vehicles with different sizes in actual conditions, and vehicle speeds are more random sometimes with overspeeding behaviour. Meanwhile, there may be special road conditions, traffic accidents and traffic controls that will affect the traffic flow. This study only considers the driver's character of each vehicle that depends on the intention strength of lane changing in perfect road conditions. In our future work, the model will be improved based on the actual road conditions, and other relevant factors will be further investigated.

\section{ACKNOWLEDGMENT}

This work was financially supported by the National Natural Science Foundation of China (Grant No. 61473028), Beijing Municipal Natural Science Foundation (Grant No. 8162031), and the National High Technology Research and Development Program of China ("863” Program) (Grant No. 2015AA124103).

\section{王江锋 ${ }^{1}$}

wangjiangfeng@bjtu.edu.cn

高畅 ${ }^{1}$

14125692@bjtu.edu.cn

朱周元 ${ }^{1}$

14125756@bjtu.edu.cn

间学东 ${ }^{1}$

xdyan@bjtu.edu.cn

1城市交通复杂系统理论与技术教育部重点实验室, 北京交通大学，北京，100044, 中国

耦合驾驶员换道意图和驾驶倾向性的多车道换道模型

摘要:

考虑不同类型驾驶员心理行为影响, 引入换道意 图和冒险系数两个定量指标, 提出耦合驾驶员换道 
意图和驾驶倾向性的多车道换道模型。基于冒险型 和保守型驾驶员心理和行为特征，分别提出相对应 的换道安全条件。数值仿真结果显示, 给出模型适 合于表述频繁换道的交通流，符合中国的驾驶员驾 驶行为。相对于STCA换道模型, 冒险系数阈值为 0.4 时，不同交通需求下所设计换道模型可提高车辆平 均速度约 $1.04 \%$; 随冒险系数阈值增加，较大交通需 求下车辆速度呈现下降趋势; 给出模型能够反映密 度、流量和速度之间关系，冒险系数会对密度和流 量关系产生显著影响。

关键词:

换道模型；换道意图；驾驶倾向；元胞自动机

\section{REFERENCES}

[1] Tang TQ, Wang YP, Yang XB. A multilane traffic flow model accounting for lane width, lane-changing and the number of lanes. Networks and Spatial Economics. 2014 Dec;14(3):465-483.

[2] Wei LY, Wang ZL, Wu RH. Research and modeling of the lane-changing behaviour on the approach. Acta Physica Sinica. 2014 Oct;63(4):1-5.

[3] Jetto K, Ez ZH, Benyoussef A. Investigation of merging and diverging cars on a multi-lane road using cellular automation model. Chinese Physics B. 2012 Jan;21(11):1-8.

[4] Veljanovska K, Bombol KM, Maher T. Reinforcement learning technique in multiple motorway access control strategy design. Promet - Traffic \& Transportation. 2010 Mar;22(2):117-123.

[5] Pei YL, Wang YG, Zhang Y. Microscopic model of automobile lane-changing virtual desire trajectory by spline curve. Promet - Traffic \& Transportation. 2010 Aug;22(3):203-208.

[6] Hoseini SMS, Vaziri M. Modelling drivers' behaviour as a crash risk reduction process. Promet - Traffic \& Transportation. 2008 Aug;20(3):139-146.

[7] Hoseini SMS. Comparison of microscopic drivers' probabilistic lane-changing models with real traffic microscopic data. Promet - Traffic \& Transportation. 2011 Mar;23(4):241-251.

[8] Tian JF, Yuan ZZ, Jia B. Cellular automaton model in the fundamental diagram approach reproducing the synchronized outflow of wide moving jams. Physics Letters A. 2012 Sep;376(44):2781-2787.

[9] Wang J, Ding JX, Shi Q. Lane-changing behaviour and its effect on energy dissipation using full velocity difference model. International Journal of Modern Physics C. 2016 Jun;27(2):1-14.

[10] Li X, Sun JQ. Studies of vehicle lane-changing to avoid pedestrians with cellular automata. Physica A. 2015 Nov;438(1):251-271.

[11] Zhao HT, Li JR, Nie C. Cellular automaton models for traffic flow considering opposite driving of an emergency vehicle. International Journal of Modern Physics C. 2015 Nov;26(7):1-12.

[12] Feng SM, Li JY, Ding N. Traffic paradox on a road segment based on a cellular automaton: impact of lane-changing behaviour. Physica A. 2015 Jun; 428(1):90-102.

[13] Nagel K, Schreckenberg M. A cellular automaton model for freeway traffic. Journal of Physique I France. 1992 Dec;2(12):2221-2229.

[14] Errampalli M, Okushima M, Akiyama T. Development of the microscopic traffic simulation model with the fuzzy logic technique. Simulation-Transactions of the Society for Modeling and Simulation International. 2013 Jan;89(1):87-101.

[15] Hua XD, Wang W, Wang H. A two-lane cellular automaton traffic flow model with the influence of driving psychology. Acta Physica Sinica. 2011 Feb;60(8):1-8.

[16] Li X, Li XG, Xiao Y. Modeling mechanical restriction differences between car and heavy truck in two-lane cellular automata traffic flow model. Physica A. 2016 Jun;451(1): 49-62.

[17] Zhu HB, Zhang NX, Wu WJ. A modified two-lane traffic model considering drivers' personality. Physica $A$. 2015 Jun;428(1):359-367.

[18] Chai C, Wong YD. Comparison of two simulation approaches to safety assessment: cellular automata and ssam. Journal of Transportation Engineering. 2015 Jan;141(6):1-12.

[19] Guzman HA, Larraga ME, Alvarez-Icaza L. A two lanes cellular automata model for traffic flow considering realistic driving decisions. Journal of cellular automata. 2015;10(1-2):65-93.

[20] Zhu HB, Zhang NX, Wu WJ. A modified two-lane traffic model considering drivers' personality. Physica A Statistical Mechanics \& Its Applications. 2015 Jun;428(1):359-367.

[21] Luo Y, Turgut D, Boeloeni L. Modeling the strategic behaviour of drivers for multi-lane highway driving. Journal of Intelligent Transportation Systems. 2015 Feb;19(1):45-62.

[22] Wang J, Cai BG, Liu J. A lane-changing behavioural preferences learning agent with its applications. Computer science and information systems. 2015 Apr;12(2):349-374.

[23] Ji X, Wu J, Zhao Y, et al. A new robust control method for active front steering considering the intention of the driver. Journal of Automobile Engineering. 2015 Mar;229(4):518-531.

[24] Bi L, Wang C, Yang X, et al. Detecting driver normal and emergency lane-changing intentions with queuing network-based driver models. International Journal of Human-Computer Interaction. 2015 Feb;31(2):139-145.

[25] Hou Y, Edara P, Sun C. Situation assessment and decision making for lane change assistance using ensemble learning methods. Expert Systems with Applications. 2015 May;42(8):3875-3882.

[26] Golbabaei F, Nejad FM, Noory AR. A microscopic analysis of speed deviation impacts on lane-changing behaviour. Transportation Planning and Technology. 2014 Jun;37(4):391-407.

[27] Wang YM, Zhou LS, Yong-Bo LV. Cellular automaton traffic flow model considering flexible safe space for lane-changing. China Journal of Highway and Transport. 2008 May; 20(5):1159-1162. 